\title{
RELAXATION OF INFINITE DIMENSIONAL VARIATIONAL AND CONTROL PROBLEMS WITH STATE CONSTRAINTS
}

\author{
By Nikolaos S. Papageorgiou*
}

\begin{abstract}
In this work we examine the question of relaxability of infinite dimensional variational problems with state constraints. We consider systems governed by multivalued evolution equations ("trajectory problem"). We start with a new, general existence result for such inclusions with nonconvex valued orientor field. Then we prove a relaxation result. Next we introduce a cost functional, which we want to minimize over the trajectories, first of the original system and then over those of the relaxed one. Using perturbed and penalized versions of the original variational problem, we show that relaxability for the system is equivalent to a well-posedness notion that we call "strong calmness". The same analysis is also carried on for semilinear systems. Now the hypotheses on the orientor field are weaker. We then show that the control problem is a special case of our trajectory problem. Finally we work an example of a distributed parameter control system.
\end{abstract}

\section{Introduction}

To establish the existence of optimal solutions in an optimal control problem, we need some kind of convexity hypothesis (recall for example the popular property $(Q)$ of Cesari [4]). This partly motivates the introduction of relaxed optimal control problems, wherein the original governing equation is replaced by its convexified analog. The nice thing about relaxed systems, is that under fairly mild hypotheses, a relaxed trajectory can be approximated arbitrarily well by an original trajectory. This, together with the facts that the relaxed problems, under very general conditions, admit optimal solutions and their value is equal to the value of the original problem, make clear the importance of the study of relaxed systems. More specifically in this paper we will study if and when the relaxation of a system introduces new better solution(s) or leaves an original optimal solution, optimal to the relaxed system (relaxability). So in some sense relaxability can be viewed as a necessary condition itself.

Since a relaxed trajectory only can be approximated arbitrarily well by an original trajectory, it is important-in particular when terminal constraints are present-to have a precise mathematical way to express the fact that the

* Research supported by N.S.F. Grants D. M.S. -8602313 and D. M. S. -8802688 .

Received October 7, 1987; Revised November 14, 1988. 
original problem is well-posed. By well-posedness, we mean that arbitrarily small perturbations of the data of the original problem, do not change drastically the minimum value of the cost functional. The first person that introduced a mathematical framework to address those questions was Clarke [6], who for this purpose defined the notion of calmness.

In this paper we investigate the relation that exists between the problem of relaxability of infinite dimensional variational problems and the property of calmness of the system. For this purpose we introduce a new, stronger version of calmness, which allows us to consider also problems with state constraints (something that is not covered by the work of Clarke [6], which deals only with finite dimensional systems). It turns out, that this stronger version of calmness, is in fact equivalent to the relaxability of the system.

To achieve a higher level of generality, we consider systems with dynamics described by differential inclusions, which include control systems, since through the "deparametrization technique", we can transform a control equation to a differential inclusion. Following Clarke's terminology (see [6]), we call this more general problem "the trajectory problem". An additional level of generality is added to our presentation, with the introduction of pointwise state constraints, something that Clarke didn't have in his problem. Finally, our systems are infinite dimensional, while Clarke [6] restricted himself to $\boldsymbol{R}^{n}$. So our work is a twofold generalization of the original work of Clarke [6]. On the one hand we allow the presence of state constraints and on the other hand we deal with infinite dimensional systems.

\section{Preliminaries}

Let $(\Omega, \Sigma)$ be a measurable space and $X$ a separable Banach space. Throughout this work we will be using the following notations:

and

$$
P_{f(c)}(X)=\{A \subseteq X: \text { nonempty, closed, (convex) }\}
$$

$$
P_{(w) k c}(X)=\{A \subseteq X: \text { nonempty, }(w-) \text { compact, (convex) }\} \text {. }
$$

A multifunction $F: \Omega \rightarrow P_{f}(X)$ is said to be measurable, if for every $x \in X$, $\omega \rightarrow d(x, F(\omega))=\inf \{\|x-z\|: z \in F(\omega)\}$ is measurable. When there is a $\sigma$-finite measure $\mu(\cdot)$, with respect to which $\Sigma$ is complete, then the above definition of measurability is equivalent to saying that $\operatorname{Gr} F=\{(\omega, x) \in \Omega \times X: x \in F(\omega)\}$ $\in \Sigma \times B(X), B(X)$ being the Borel $\sigma$-field of $X$ (graph measurability). By $S_{F}^{p}$ $(1 \leqq p \leqq \infty)$, we will denote the set of measurable selectors of $F(\cdot)$ that belong in the Lebesgue-Bochner space $L^{p}(X)$ i. e. $S_{F}^{p}=\left\{f \in L^{p}(X): f(\omega) \in F(\omega) \mu\right.$-a. e. $\}$. This set may be empty. It is nonempty if and only if $\omega \rightarrow \inf \{\|z\|: z \in F(\omega)\} \in L_{+}^{p}$. In particular this is the case if $\omega \rightarrow|F(\omega)|=\sup \{\|z\|: z \in F(\omega)\} \in L_{+}^{p}$, in which case $F(\cdot)$ is said to be $L^{p}$-integrably bounded.

Let $Y, Z$ be Hausdorff topological spaces and $G: Y \rightarrow 2^{Z} \backslash\{\varnothing\}$ a multifunction. We say that $G(\cdot)$ is upper semicontinuous (u.s.c.) (resp. lower semicontinuous 
(l.s. c.)), if for all $V \leqq Z$ open, we have that $G^{+}(V)=\{y \in Y: G(y) \subseteq V\}$ (resp. $\left.G^{-}(V)=\{y \in Y: G(y) \cap V \neq \varnothing\}\right)$ is open in $Y$. Also if $Z$ is a completely regular space on the space $M(Z)$ of bounded Radon measures on $Z$ we can define the narrow topology to be the weak topology $w\left(M(Z), C_{b}(Z)\right)$, where $C_{b}(Z)$ are the bounded continuous functions on $Z$.

Finally if $\Omega=T=[0, b]$ and $f \in L^{1}(X)$, the weak norm of $f(\cdot)$ is defined as follows :

$$
\|f\|_{w}=\sup \left\{\left\|\int_{t}^{t^{\prime}} f(s) d s\right\|: t^{\prime}, t \in T\right\} .
$$

Convergence of a sequence in this norm, implies weak convergence in $L^{1}(X)$. For the converse additional hypotheses are needed.

\section{A relaxation result.}

Let $T=[0, b], H$ a separable Hilbert space and $X$ a subspace of $H$ carrying the structure of a separable, reflexive Banach space and continuously and densely embedded into $H$ (i.e. $X c_{\rightarrow} H$ ). Having $H$ as our pivot space, we have $X \hookrightarrow H \hookrightarrow X^{*}$ and we assume that the embeddings are also compact. By $\|\cdot\|$ (resp. $|\cdot|,\|\cdot\|_{*}$ ), we will denote the norm of $X$ (resp. of $H, X^{*}$ ). Also by $\langle\cdot, \cdot\rangle$ we will denote the duality brackets for the pair $\left(X, X^{*}\right)$ and by $(\cdot, \cdot)$ the inner product in $H$. The two are compatible in the sense that $\left.\langle\cdot, \cdot\rangle\right|_{X \times H}=(\cdot, \cdot)$.

We consider the following two multivalued systems:

The "original system":

$$
\left\{\begin{array}{l}
\dot{x}(t)+A(t, x(t)) \in F(t, x(t)) \text { a. e. } \\
x(0)=x_{0}
\end{array}\right\}
$$

and the "relaxed system":

$$
\left\{\begin{array}{l}
\dot{x}(t)+A(t, x(t)) \in \overline{\operatorname{conv}} F(t, x(t)) \text { a. e. } \\
x(0)=x_{0}
\end{array}\right\}
$$

Our goal in this section, is to establish a relation between the sets of trajectories of the above two multivalued systems.

We will achieve this, by first establishing the existence of strong solutions for problem (*). This result is of independent interest and extends earlier existence results for differential inclusions obtained by Kisielewicz [11], PavelVrabie [19] and Papageorgiou [14]. The interesting feature of our existence result is the nonconvexity of the orientor field $F(\cdot, \cdot)$. To prove this general existence theorem, we need the following set of hypotheses. Here $X_{w}$ denotes the space $X$ with the weak topology. 
$H(A): A: T \times X \rightarrow X^{*}$ is an operator s.t.

(1) $t \rightarrow A(t, x)$ is measurable,

(2) $x \rightarrow A(t, x)$ is sequentially continuous from $X_{w}$ into $X_{w}^{*}$ and monotone,

(3) $\langle A(t, x), x\rangle \geqq c_{1}\|x\|^{p}$ a. e., with $c_{1}>0$ and $1<p<\infty$,

(4) $\|A(t, x)\|_{*} \leqq h(t)+c_{2}\|x\|^{p-1}$ a. e., with $h(\cdot) \in L^{q} 1 / p+1 / q=1$ and $c_{2}>0$.

$H(F): \quad F: T \times X \rightarrow P_{f}\left(X^{*}\right)$ is a multifunction s.t.

(1) $(t, x) \rightarrow F(t, x)$ is graph measurable and $|F(t, x)| \leqq a(t)$ a.e. with $a(\cdot) \in L^{q}$,

(2) $x \rightarrow F(t, x)$ is 1. s.c. from $X_{w}$ into $X^{*}$.

By a solution of $\left(^{*}\right)$ we understand an absolutely continuous function

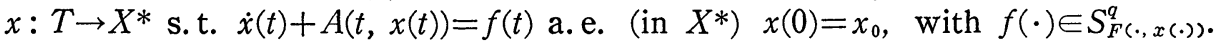
By $W(T)$ we will denote the following Banach space: $W(T)=\left\{x(\cdot) \in L^{p}(X)\right.$ : $\left.\dot{x}(\cdot) \in L^{q}\left(X^{*}\right)\right\}$. Recall that $W(T) \subseteq C(T, H)$ (see Lions [12]).

THEOREM 3.1. If hypotheses $H(A)$ and $H(F)$ hold and $x_{0} \in X$, then (*) admits a solution belonging in $W(T)$.

Proof. Consider $K \subseteq C(T, H)$ defined by:

$$
\begin{array}{r}
K=\{y \in C(T, H): \dot{y}(t)+A(t, y(t))=g(t) \text { a.e. } \\
\left.\quad \text { in } X^{*}, y(0)=x_{0},\|g(t)\|_{*} \leqq a(t) \text { a. e. }\right\} .
\end{array}
$$

From theorem 4.2, p. 166 of Barbu [2], we know that $\varnothing \neq K \subseteq W(T)$.

We will show that $K$ is a compact, metrizable subset of $C\left(T, X_{w}\right)$.

First we will determine an a priori bound for the elements in $K$. So for $x \in K$, we have:

$$
\begin{aligned}
& \langle\dot{x}(t), x(t)\rangle=\langle-A(t, x(t)), x(t)\rangle+\langle g(t), x(t)\rangle \text { a.e. } \\
& \Rightarrow \frac{d}{d t}\langle x(t), x(t)\rangle=2\langle-A(t, x(t)), x(t)\rangle+2\langle g(t), x(t)\rangle \text { a.e. }
\end{aligned}
$$

But recall that $\langle x(t), x(t)\rangle=(x(t), x(t))$. So we get:

$$
\frac{d}{d t}(x(t), x(t))=\frac{d}{d t}|x(t)|^{2}=2\langle-A(t, x(t)), x(t)\rangle+2\langle g(t), x(t)\rangle .
$$

Because of hypotheses $H(A)$ (1) and (2), we see that for every $y \in X$, $(t, x) \rightarrow\langle A(t, x), y\rangle$ is a Caratheodory function (i. e. measurable in $t$, continuous in $x) \Rightarrow(t, x) \rightarrow\langle A(t, x), y\rangle$ is jointly measurable $\Rightarrow t \rightarrow\langle A(t, x(t)), x(t)\rangle$ is measurable and because of hypothesis $H(A)(4)$, is integrable. So integrating both sides of the last equality above we get that:

$$
|x(t)|^{2}-\left|x_{0}\right|^{2}+2 \int_{0}^{t}\langle A(s, x(s)), x(s)\rangle d s=2 \int_{0}^{t}\langle g(s), x(s) d s .
$$

Using the coercivity hypothesis $H(A)(3)$ and Hölder's inequality, we get: 


$$
|x(t)|^{2}+2 c_{1} \int_{0}^{t}\|x(s)\|^{p} d s \leqq\left|x_{0}\right|^{2}+2\left(\int_{0}^{t}\|g(s)\|_{*}^{q} d s\right)^{1 / q}\left(\int_{0}^{t}\|x(s)\|^{p} d s\right)^{1 / p}
$$

From Cauchy's inequality, we have:

$$
\left(\int_{0}^{t}\|g(s)\|_{*}^{q}\right)^{1 / q} \cdot\left(\int_{0}^{t}\|x(s)\|^{p} d s\right)^{1 / p} \leqq\left(1 / q \varepsilon^{q}\right) \int_{0}^{t}\|g(s)\|_{*}^{q} d s+\left(\varepsilon^{p} / p\right) \int_{0}^{t}\|x(s)\|^{p} d s .
$$

Take $\varepsilon=\left(p c_{2} / 2\right)^{1 / p}$. Then for all $t \in T$ we have:

$$
|x(t)|^{2}+c_{1} \int_{0}^{t}\|x(s)\|^{p} d s \leqq|x(0)|^{2}+\left(2 / q\left(\frac{p c_{2}}{2}\right)\right)^{p / q}\|a\|_{q}^{q}=M
$$

Having this estimate we can now get the relative sequential compactness of $K$ in $C\left(T, X_{w}\right)$. To this end consider $Z \cong L^{q}\left(X^{*}\right)$ defined by:

$$
Z=\left\{y \in L^{q}\left(X^{*}\right): \int_{A} y(s) d s \in X \text { for all } A \subseteq T \text { Lebesgue measurable }\right\} \text {. }
$$

Clearly $Z$ is a linear subspace of $L^{q}\left(X^{*}\right)$. Let $\left\{y_{n}\right\}_{n \geq 1} \subseteq Z$ s.t. $y_{n} \stackrel{s}{\rightarrow} y$ in $L^{q}\left(X^{*}\right)$. Then for every $x \in X$, we have:

$$
\begin{aligned}
& \int_{0}^{b}\left\langle\chi_{A}(s) x, y_{n}(s)\right\rangle d s \longrightarrow \int_{0}^{b}\left\langle\chi_{A}(s) x, y(s)\right\rangle d s \\
& \Rightarrow\left\langle x, \int_{A} y_{n}(s) d s\right\rangle \longrightarrow\left\langle x, \int_{A} y(s) d s\right\rangle .
\end{aligned}
$$

Since $X \subseteq H \subset X^{*}$, with all embeddings continuous and dense, we deduce that:

$$
\int_{A} y_{n}(s) d s \stackrel{w}{\longrightarrow} \int_{A} y(s) d s \text { in } X \text {. }
$$

Because $X$ is reflexive, is weakly sequentially complete. So $\int_{A} y(s) d s \in X$ for all $A \subseteq T$ Lebesgue measurable $\Rightarrow y \in Z \Rightarrow Z$ is closed in $L^{q}\left(X^{*}\right) \Rightarrow Z$ is a separable, reflexive Banach space for the relative $L^{q}\left(X^{*}\right)$-norm topology.

Now let $A \subseteq T$ be Lebesgue measurable and consider the linear operator $K(A): Z \rightarrow X$ defined by :

$$
K(A)(y)=\int_{0}^{b} \chi_{A}(t) y(t) d t
$$

For every $x^{*} \in X^{*}, y \rightarrow\left\langle x^{*}, K(A)(y)\right\rangle$ is a continuous linear functional on $Z$. So there exists $g\left(x^{*}\right)(\cdot) \in L^{p}(X)$ s.t.

$$
\begin{aligned}
\left\langle x^{*}, K(A)(y)\right\rangle & =\int_{A}\left\langle g\left(x^{*}\right)(s), y(s)\right\rangle d s \\
& \leqq\left(\int_{A}\left\|g\left(x^{*}\right)(s)\right\|^{p} d s\right)^{1 / p} \cdot\|y\|_{q}
\end{aligned}
$$


On the other hand, we have that:

$$
x(t+h)-x(t)=\int_{t}^{t+h} \dot{x}(s) d s
$$

So for $x^{*} \in X^{*}$, we have :

$$
\left\langle x^{*}, x(t+h)-x(t)\right\rangle \leqq\left(\int_{t}^{t+h}\left\|g\left(x^{*}\right)(s)\right\|^{p} d s\right)^{1 / p} \cdot\|\dot{x}\|_{q}
$$

We claim that there exists $M_{0}>0$ s.t. for all $x \in K,\|\dot{x}\|_{q} \leqq M_{0}$. To this end, let $v \in L^{p}(X)$. Then for $x \in K$, we have:

$$
\begin{aligned}
\int_{0}^{b}\langle\dot{x}(t), v(t)\rangle d t & =\int_{0}^{b}\langle-A(t, x(t))+g(t), v(t)\rangle d t \\
& \leqq \int_{0}^{b}\left(\left\|A\left(t, x(t)\left\|_{*}+\right\| g(t) \|_{*}\right) \cdot\right\| v(t) \| d t .\right.
\end{aligned}
$$

Applying Hölder's and Minkowski's inequalities and using hypothesis $H(A)$ (4), we get:

$$
\begin{aligned}
& \int_{0}^{b}\left(\|A(t, x(t))\|_{*}+\|g(t)\|_{*}\right) \cdot\|v(t)\| d t \\
\leqq & {\left[\int_{0}^{b}\left(\|A(t, x(t))\|_{*}^{q}+\|g(t)\|_{*}^{q} d t\right]^{1 / q} \cdot\|v\|_{p}\right.} \\
\leqq & {\left[\left(\int_{0}^{b}\|A(t, x(t))\|_{*}^{q}\right)^{1 / q}+\left(\int_{0}^{b}\|g(t)\|_{*}^{q}\right)^{1 / q}\right] \cdot\|v\|_{p} } \\
\leqq & {\left[\left(\int_{0}^{b}\left(h(t)+c_{2}\|x(t)\|^{p-1}\right)^{p / p-1} d t\right)^{1 / q}+\|g\|_{q}\right] \cdot\|v\|_{p} . }
\end{aligned}
$$

But from estimate (1) above, we know that $\|x\|_{p}^{p} \leqq M / c_{1}$. Hence from (2) we get:

$$
((\dot{x}, v)) \leqq\left[\|h\|_{q}^{q}+c_{2}\left(M / c_{1}\right)^{1 / q}+\|g\|_{q}\right] \cdot\|v\|_{p}
$$

where $((\cdot, \cdot))$ denotes the duality brackets for the pair $\left\langle L^{p}(X), L^{q}\left(X^{*}\right)=\left(L^{p}(X)\right)^{*}\right\rangle$. Therefore finally we have:

$$
\sup \left\{((\dot{x}, v)):\|v\|_{p} \leqq 1\right\}=\|\dot{x}\|_{q} \leqq M_{0}
$$

for all $x \in K$ and with $M_{0}=\|h\|_{q}^{q}+c_{2}\left(M / c_{1}\right)^{1 / q}+\|g\|_{q}$ and so the claim about the $L^{q}\left(X^{*}\right)$-boundedness of $\{\dot{x}(\cdot): x \in K\}$ follows.

So now we have:

$$
\left\langle x^{*}, x(t+h)-x(t)\right\rangle \leqq\left(\int_{t}^{t+h}\left\|g\left(x^{*}\right)(s)\right\|^{p} d s\right)^{1 / p} \cdot M_{0}
$$

$\Rightarrow K$ is weakly equicontinuous.

Furthermore, once again from estimate (1) and recalling that in a reflexive Banach space, bounded sets are relatively $w$-compact, we deduce that for all 
$t \in T, \overline{\{x(t): x \in K}\}^{w} \in P_{w k}(X)$.

Invoking the Arzela-Ascoli theorem, we deduce that $K$ is relatively sequentially compact in $C\left(T, X_{w}\right)$.

Next we will show that $K$ is sequentially closed in $C\left(T, X_{w}\right)$. From the Dunford-Pettis compactness criterion and by passing to a subsequence if necessary, we may assume that $g_{n} \stackrel{w}{\rightarrow} g$ in $L^{q}\left(X^{*}\right)$. Using the dominated convergence theorem, we get that:

$$
\int_{0}^{t} A\left(s, x_{n}(s)\right) d s \stackrel{w}{\longrightarrow} \int_{0}^{t} A(s, x(s)) d s
$$

while we also have $\int_{0}^{t} g(s) d s \stackrel{w}{\longrightarrow} \int_{0}^{t} g(s) d s$. Therefore in the limit we get:

$$
\begin{aligned}
& x(t)=x_{0}-\int_{0}^{t} A(s, x(s)) d s+\int_{0}^{t} g(s) d s \\
& \Rightarrow \dot{x}(t)+A(t, x(t))=g(t) \text { a. e., } x(0)=x_{0} \\
& \Rightarrow x \in K .
\end{aligned}
$$

So indeed $K$ is sequentially closed, hence sequentially compact in $C\left(T, X_{w}\right)$. It is also metrizable since $K \cong C\left(T, B\left(0, M_{1}\right)_{w}\right)$, with $M_{1}=\sup _{t \in T}\|x(t)\|$ and $B\left(0, M_{1}\right)_{w}$ is the $M_{1}$-ball in $X$ with the weak topology. Since $B\left(0, M_{1}\right)_{w}$ is compact, metrizable, then so is the compact-open topology on $C\left(T, B\left(0, M_{1}\right)_{w}\right)$.

Let $\hat{K}=\overline{\operatorname{conv}} K$. This is still compact in $C\left(T, X_{w}\right)$. Consider the multifunction $L: \hat{K} \rightarrow 2^{L^{1}\left(X^{*}\right)}$ defined by:

$$
L(x)=S_{F(\cdot, x(\cdot))}^{1}
$$

Using hypothesis $H(F)(2)$ and theorem 4.1 of [16], we get that $L(\cdot)$ is 1.s.c. Apply Fryszkowski's selection theorem [9], to get $p: \hat{K} \rightarrow L^{1}\left(X^{*}\right)$ continuous s.t. $p(x) \in L(x)$ for all $x \in \hat{K}$. Then consider the evolution equation

$$
\left\{\begin{array}{l}
\dot{y}(t)+A(t, y(t))=p(x)(t) \text { a. e. } \\
y(0)=x_{0}
\end{array}\right\}(*)^{\prime}
$$

Again from Barbu [2], we know that this has a unique solution in $W(T)$. Let $q: \hat{K} \rightarrow \hat{K}$ be the map that to each $x \in \hat{K}$ assigns the unique solution of $\left({ }^{*}\right)^{\prime}$. We claim that this map is continuous for the relative $C\left(T, X_{w}\right)$-topology on $\hat{K}$. So let $x_{n} \rightarrow x$ in $\hat{K}$. Then by setting $y_{n}=q\left(x_{n}\right) \in \hat{K}$ and by passing to a subsequence if necessary, we may assume that $y_{n} \rightarrow y$ in $\hat{K}$. So we have:

$$
\int_{0}^{t} A\left(s, y_{n}(s)\right) d s \stackrel{w}{\longrightarrow} \int_{0}^{t} A(s, y(s)) d s
$$

and

$$
\int_{0}^{t} p\left(x_{n}\right)(s) d s \longrightarrow \int_{0}^{t} p(x)(s) d s
$$




$$
\begin{aligned}
& \Rightarrow y(t)=x_{0}-\int_{0}^{t} A(s, y(s)) d s+\int_{0}^{t} p(x)(s) d s \\
& \Rightarrow y=q(x) \\
& \Rightarrow q(\cdot) \text { is continuous on } \hat{K} \text { with the } C\left(T, X_{w}\right) \text {-topology. }
\end{aligned}
$$

Apply Tichonoff's fixed point theorem, to get $x \in \hat{K}$ s.t. $x=q(x)$. This is the desired solution of $(*)$.

Now we are ready to compare the solution sets of $\left(^{*}\right)$ and $(* *)$. To achieve this we need the following stronger set of hypotheses:

$H(A)_{1}: A: T \times X \rightarrow X^{*}$ is an operator s.t.

(1) $t \rightarrow A(t, x)$ is measurable,

(2) $x \rightarrow A(t, x)$ is sequentially weakly continuous and monotone,

(3) $\langle A(t, x), x\rangle \geqq c_{1}\|x\|^{p}$ a. e., with $c_{1}>0,1<p<\infty$,

(4) $\|A(t, x)\|_{*} \leqq h(t)+c_{2}\|x\|^{p-1}$, with $h(\cdot) \in L^{q}, 1 / p+1 / q=1, c_{2}>0$.

$H(F)_{1}: \quad F: T \times H \rightarrow P_{f}(H)$ is a multifunction s.t. $F(t, x)=p(t, x, U(t))$, where:

(1) $p: T \times H \times Z \rightarrow H$ with $Z$ a separable Banach space and:

i) $t \rightarrow p(t, x, z)$ is measurable,

ii) $\left|p(t, x, z)-p\left(t, x^{\prime}, z\right)\right| \leqq k(t)\left|x-x^{\prime}\right|$ a. e. with $k(\cdot) \in L_{+}^{1}$,

iii) $(x, z) \rightarrow p(t, x, z)$ is continuous from $H \times Z_{w}$ into $H_{w}$,

iv) $|p(t, x, z)| \leqq a(t)$ a. e. with $a(\cdot) \in L^{q}$.

(2) $U: T \rightarrow P_{f c}(Z)$ is an integrably bounded multifunction s.t. $U(t) \subseteq W$ a. e., with $W \in P_{w k c}(Z)$.

Note that $F(t, x)=p(t, x, U(t)) \in P_{w k}(H)$ and so from the Krein-Smulian theorem, we have that $\overline{\operatorname{conv}} F(t, x) \in P_{w k c}(H)$.

We will denote the solution set of $\left(^{*}\right)$ by $P\left(x_{0}\right)$ and the solution set of $\left(^{* *}\right)$ by $P_{r}\left(x_{0}\right)$. We have the following result relating those two sets.

Theorem 3.2. If hypotheses $H(A)_{1}$ and $H(F)_{1}$ hold and $x_{0} \in X$, then $P_{r}\left(x_{0}\right)$ is nonempty, sequentially compact in $C\left(T, X_{w}\right)$ and $\overline{P\left(x_{0}\right)}=P_{r}\left(x_{0}\right)$, the closure taken in $C\left(T, X_{w}\right)$.

Proof. The nonemptiness of $P_{r}\left(x_{0}\right)$ follows from theorem 3.1.

Let $M(Z)$ be the set of bounded Borel measures on $Z$ and denote by $M_{+}^{1}(Z)$ the probability measures on $Z$. Define $\Sigma(t)=\left\{\lambda \in M_{+}^{1}(Z): \lambda(U(t))=1\right\}$. From theorem V-2 of Castaing-Valadier [4], we know that $\Sigma(\cdot)$ is measurable. Set $F_{r}(t, x)=\left\{\int_{W} p(t, x, z) \lambda(d z): \lambda \in \Sigma(t)\right\}$. We claim that $F_{r}(t, x)=\overline{\operatorname{conv}} F(t, x)$. First note that $F_{r}(t, x)$ is convex. Also if $x_{n} \in F_{r}(t, x), n \geqq 1$ and $x_{n} \rightarrow x$ we have $x_{n}=\int_{W} p(t, x, z) \lambda_{n}(d z), \lambda_{n} \in \Sigma(t)$. But $\Sigma(t) \leqq M_{+}^{1}\left(W_{w}\right)$ and the latter is a compact. 
Polish space with the narrow (weak)-topology since $W_{w}$ is compact, Polish. So we may assume that $\lambda_{n} \stackrel{w}{\rightarrow} \lambda \in \Sigma(t)$ (since $\lim \lambda_{n}(U(t))=1 \leqq \lambda(U(t))$ ). So for every $v \in H, \quad\left(v, x_{n}\right)=\int_{W}(v, p(t, x, z)) \lambda_{n}(d z) \rightarrow \int_{W}(v, p(t, x, z)) \lambda(d z) \Rightarrow\left(v, \int_{W} p(t, x, z) \lambda(d z)\right)$ $\lambda \in \Sigma(t)$, for all $v \in H \Rightarrow x=\int_{W} p(t, x, z) \lambda(d z), \lambda \in \Sigma(t) \Rightarrow x \in F_{r}(t, x) \Rightarrow F_{r}(t, x)$ is closed. Also note that $F(x, t) \leqq F_{r}(t, x)$ (just take $\lambda=\delta_{u}, u \in U(t)$ ). Hence $\overline{\operatorname{conv}} F(t, x) \subseteq F_{r}(t, x)$. On the other hand given $\lambda \in \Sigma(t)$, from a well known property of probability measures, we can find $\lambda_{n}=\sum_{k=1}^{N_{n}} a_{k} \delta_{u_{k}}$ where $a_{k} \geqq 0$, $\sum_{k=1}^{N} a_{k}=1$ and $u_{k} \in U(t)$, s. t. $\lambda_{n} \stackrel{w}{\rightarrow} \lambda$. So $\int_{W} p(t, x, z) \lambda_{n}(d z) \stackrel{w=1}{\rightarrow} \int_{W} p(t, x, z) \lambda(d z)$. But $\int_{W} p(t, x, z) \lambda_{n}(d z)=\sum_{k=1}^{N} p\left(t, x, u_{k}\right) \in \operatorname{conv} F(t, x)$. So $F_{r}(t, x) \cong \overline{\operatorname{conv}} F(t, x) \Rightarrow F_{r}(t, x)$ $=\overline{\operatorname{conv}} F(t, x)$ as claimed.

From the definition of $F(t, x)$ we have:

$$
\begin{aligned}
G r F & =\{(t, x, y) \in T \times H \times H: y \in F(t, x)\} \\
& =\{(t, x, y) \in T \times H \times H: y=p(t, x, u), u \in U(t)\}
\end{aligned}
$$

Set $\eta(t, x, y, u)=y-p(t, x, u)$ and $\theta(t, u)=d(u, U(t))$. Then we have:

$$
G r F=\operatorname{proj}_{T \times H \times H}[(t, x, y, u): \eta(t, x, y, u)=0, \theta(t, u)=0] .
$$

Note that both $\eta$ and $\theta$ are $B(T) \times B(H) \times B(H) \times B(W)$-measurable mappings. Also from corollary 2.4 of Edgar [8], we know that $B(Z)=B\left(Z_{w}\right) \Rightarrow B(W)=$ $B(Z) \cap W=B\left(Z_{w}\right) \cap W=B\left(W_{w}\right)$ (where $W_{w}$ denotes the set $W$ with the weak topology). But recall that $W_{w}$ is compact, Polish. So from the Arsenin-Novikov theorem (see Saint-Beuve [21]), we get that:

$$
G r F \in B(T) \times B(H) \times B(H) .
$$

Hence $F(\cdot, \cdot)$ is graph measurable and so we can apply theorem 2 of Chuong [7] and get that $S_{F(\cdot, x(\cdot))}^{1}$ is dense $S_{\overline{c o n v} F(\cdot, x(\cdot))}^{1}=S_{F_{r}}^{1}(\cdot, x(\cdot))$ for the weak norm $\|\cdot\|_{w}$. So if $x(\cdot) \in P_{r}\left(x_{0}\right)$ and $n \geqq 1$ we can find $f_{n} \in S_{F(\cdot, x(\cdot))}^{1}$ s.t. $\left\|f_{n}-f\right\|_{w}$ $\rightarrow 0$, where $f(\cdot) \in S_{F_{r}(\cdot, x(\cdot))}^{1}=S^{1} \overline{\text { conv } F(\cdot, x(\cdot))}$ s. t. $\dot{x}(t)+A(t, x(t))=f(t)$ a. e.

Consider the multifunction $L_{n}: T \rightarrow 2^{Z} \backslash\{\varnothing\}, n \geqq 1$, defined by:

$$
L_{n}(t)=\left\{u \in U(t): f_{n}(t)=p(t, x(t), u)\right\} .
$$

Clearly $L_{n}(t) \neq \varnothing$ for all $t \in T$. Let $\left\{x_{m}\right\}_{m \geq 1}$ be dense in $X$ and define $h_{m}^{n}(t, u)=\left(x_{m}, f_{n}(t)-p(t, x(t), u)\right)$. From hypothesis $H(F)_{1}$, it is easy to see that $(t, u) \rightarrow h_{m}^{n}(t, u)$ is a Caratheodory function, hence jointly measurable. So for every $m \geqq 1\left\{(t, u) \in T \times Z: h_{m}^{n}(t, u)=0\right\} \in B(T) \times B(Z)$. Observe that: 


$$
\begin{aligned}
& G r L_{n}=\bigcap_{m \geqq 1}\left\{(t, u): h_{m}^{n}(t, u)=0\right\} \cap G r U \\
& G r L_{n} \in B(T) \times B(Z) .
\end{aligned}
$$

So we can apply Aumann's selection theorem (see Saint-Beuve [20], theorem 3 ) and get $u_{n}: T \rightarrow Z$ measurable s.t. $u_{n}(t) \in L_{n}(t)$ a. e. So we have: $f_{n}(t)=$ $p\left(t, x(t), u_{n}(t)\right)$ a. e., with $u_{n}(\cdot) \in S_{U}^{1}$.

Let $y_{n}(\cdot)$ be the unique strong solution of the original system corresponding to the forcing term $p\left(t, y_{n}(t), u_{n}(t)\right)$. So $y_{n}(\cdot) \in P\left(x_{0}\right)$ and since from the proof of theorem 3.1 we have that $P\left(x_{0}\right)$ is relatively sequentially compact in $C\left(T, X_{w}\right)$, by passing to a subsequence if necessary, we may assume that $y_{n} \rightarrow y$ in $C\left(T, X_{w}\right)$. Then for all $n \geqq 1$, we have:

$$
\begin{aligned}
& \frac{d}{d t}\left|x(t)-y_{n}(t)\right|^{2}=2\left\langle x(t)-\dot{y}_{n}(t), x(t)-y_{n}(t)\right\rangle \\
& =2\left\langle-A(t, x(t))+f(t)+A\left(t, y_{n}(t)\right)-p\left(t, y_{n}(t), u_{n}(t)\right), x(t)-y_{n}(t)\right\rangle \text { a. e. } \\
& \leqq 2\left(f(t)-p\left(t, y_{n}(t), u_{n}(t)\right), x(t)-y_{n}(t)\right\rangle \text { a. e. } \\
& \quad \quad \text { since } A(t, \cdot) \text { is monotone) } \\
& =2\left(f(t)-p\left(t, x(t), u_{n}(t)\right)+p\left(t, x(t), u_{n}(t)\right)-p\left(t, y_{n}(t), u_{n}(t)\right), x(t)-y_{n}(t)\right\rangle \\
& \Rightarrow\left|x(t)-y_{n}(t)\right|^{2} \leqq \int_{0}^{t} 2\left(f(s)-f_{n}(s), x(s)-y_{n}(s)\right) d s+\int_{0}^{t} k(s)\left|x(s)-y_{n}(s)\right|^{2} d s .
\end{aligned}
$$

Since $y_{n} \rightarrow y$ in $C\left(T, X_{w}\right) \Rightarrow y_{n}(t) \stackrel{w}{\rightarrow} y(t)$ in $X$ and since $X \hookrightarrow H$ compactly $y_{n}(t) \stackrel{s}{\rightarrow} y(t)$ in $H$. Furthermore recall that $f_{n} \stackrel{\|\cdot\|_{w}}{\rightarrow} f$ and so $f_{n} \stackrel{w}{\rightarrow} f$ in $L^{1}(H)$. Thus in the limit we get:

$$
|x(t)-y(t)|^{2} \leqq \int_{0}^{t} k(s)|x(s)-y(s)|^{2} d s .
$$

Invoking Gronwall's inequality we conclude that $x=y \Rightarrow x \in \overline{P\left(x_{0}\right)} \Rightarrow P_{r}\left(x_{0}\right)$ $=\overline{P\left(x_{0}\right)}$ the closure in $C\left(T, X_{w}\right)$.

Q.E.D.

Remark. From the above proof it is easy to see that instead of the Lipschitz hypothesis on $p(t, \cdot, u)$ we could have made a dissipativity hypothesis. In general from the theory of differential inclusions (see Aubin-Cellina [1]), we know that simple continuity of the orientor field is not enough to have a relaxation result. In the context of control systems, the relaxation result just proved, tells us that essentially (i.e. within closure), we can have the same attainable set by economizing in the controls.

\section{Relaxability of variational problems and calmness}

In this section we impose state constraints and we introduce a cost functional, which we try to minimize first over the set of trajectories of the original system 
and then over the set of trajectories of the relaxed one. Our goal is to derive necessary and sufficient conditions for the two variational problems to have the same value. In this process, we also introduce our stronger version of the notion of calmness due to Clarke [6].

So let $f: C(T, H) \rightarrow \boldsymbol{R}$ and $k: H \times H \rightarrow \boldsymbol{R}$ be two continuous functions. The cost functional is:

$$
J(x)=f(x)+k(x(0), x(b)) .
$$

We want to minimize $J(\cdot)$, first over the trajectories of

$$
\left\{\begin{array}{l}
\dot{x}(t)+A(t, x(t)) \in F(t, x(t)) \text { a. e. } \\
x(t) \in K(t)
\end{array}\right\} \quad(*)_{1}
$$

and then over the trajectories of

$$
\left\{\begin{array}{l}
\dot{x}(t)+A(t, x(t)) \in \overline{\operatorname{conv}} F(t, x(t)) \text { a. e. } \\
x(t) \in K(t)
\end{array}\right\}
$$

Denote by $m$ the value of the first optimization problem and by $m_{r}$ the value of the second one. We want to compare them ("relaxability problem").

We will need the following hypothesis concerning the state constraint multifunction.

$H(K): \quad K: T \rightarrow P_{f}(H)$ is measurable and $K(0) \leqq X$ is bounded.

Given the a priori bounds for the trajectories of $(*)_{1}$ and $(* *)_{1}$, there is no loss of generality in assuming that $K(\cdot)$ is integrably bounded.

We will approach the relaxability problem by considering perturbed and penalized versions of the original variational problem and examining the behavior of the values of those problems as the perturbation and penalization respectively go to zero. So the perturbed problem has the following form:

$$
\left\{\begin{array}{l}
f(x)+k(x(0), x(b)) \rightarrow \inf \\
\text { s. t. } \dot{x}(t)+A(t, x(t)) \in F(t, x(t)) \text { a. e. } \\
\int_{0}^{b} d_{H}(x(t), K(t)) d t \leqq \varepsilon, x(0) \in K(0)
\end{array}\right\}(*)_{\varepsilon}
$$

while the penalized problem is the following one:

$$
\left\{\begin{array}{l}
f(x)+k(x(0), x(b))+(1 / \varepsilon) \int_{0}^{b} d_{H}(x(t), K(t)) d t \rightarrow \inf \\
\text { s.t. } \dot{x}(t)+A(t, x(t)) \in F(t, x(t)) \text { a. e. } x(0) \in K(0)
\end{array}\right\} \quad(*)_{\varepsilon}^{\prime}
$$

Here $d_{H}(\cdot, K(t))$ denotes the distance fnnction in $H$ from the set $K(t) \cong H$. We will denote the value of $\left({ }^{*}\right)_{\varepsilon}$ by $m(\varepsilon)$ and the value of $(*)_{\varepsilon}^{\prime}$ by $m^{\prime}(\varepsilon)$. We will assume that they are finite and set $m(0)=m^{\prime}(0)=m$. Throughout this section we will assume that $\left({ }^{*}\right)_{1}$ (hence $(* *)_{1}$ too) admits solutions. Nagumo type conditions that will guarantee this for particular cases can be found in Pavel [18] 
(semilinear systems) and Papageorgiou-Avgerinos [17] (nonlinear autonomous systems).

THEOREM 4.1. If hypotheses $H(A), H(F)$ and $H(K)$ hold then $m(\cdot)$ is right continuous at 0 if and only if $m^{\prime}(\cdot)$ is right continuous at 0.

Proof. $\Downarrow$ : Let $\left\{x_{n}\right\}_{n \geqslant 1} \leqq C(T, H)$ be trajectories of the evolution inclusion $\dot{x}_{n}(t)+A\left(t, x_{n}(t)\right) \in F\left(t, x_{n}(t)\right)$ a. e. and s.t.

$$
f\left(x_{n}\right)+k\left(x_{n}(0), x_{n}(b)\right)+n \int_{0}^{b} d_{H}\left(x_{n}(t), K(t)\right) d t \leqq m^{\prime}(1 / n)+1 / n .
$$

From the above inequality we get that $\left\{n \int_{0}^{b} d_{H}\left(x_{n}(t), K(t)\right) d t\right\}_{n \geqq 1}$ is bounded. So there exists $M>0$ s.t.

$$
\begin{aligned}
& n \int_{0}^{b} d_{H}\left(x_{n}(t), K(t)\right) d t \leqq M \text { for all } n \geqq 1 \\
& \Rightarrow \int_{0}^{b} d_{H}\left(x_{n}(t), K(t)\right) d t \leqq M / n \\
& \Rightarrow m(M / n) \leqq m^{\prime}(1 / n)+1 / n \\
& \Rightarrow \lim _{n \rightarrow \infty} m(M / n)=m(0)=m^{\prime}(0) \leqq \lim _{n \rightarrow \infty} m^{\prime}(1 / n) .
\end{aligned}
$$

Since $m^{\prime}(1 / n) \leqq m^{\prime}(0)$ for all $n \geqq 1$, we conclude that $m^{\prime}(\cdot)$ is right continuous at zero.

爪: Again let $\left\{x_{n}\right\}_{n \geqq 1} \leqq C(T, H)$ be s.t. $\dot{x}_{n}(t)+A\left(t, x_{n}(t)\right) \in F\left(t, x_{n}(t)\right)$ a.e., $\int_{0}^{b} d_{H}\left(x_{n}(t), K(t)\right) d t \leqq 1 / n^{2}$ and $f\left(x_{n}\right)+k\left(x_{n}(0), x_{n}(b)\right) \leqq m\left(1 / n^{2}\right)+1 / n, n \geqq 1$. Then $n \int_{0}^{b} d_{H}\left(x_{n}(t), K(t)\right) d t \leqq 1 / n$ and so we have:

$$
\begin{aligned}
& f\left(x_{n}\right)+k\left(x_{n}(0), x_{n}(b)\right)+n \int_{0}^{b} d_{H}\left(x_{n}(t), K(t)\right) d t \leqq m\left(1 / n^{2}\right)+2 / n \\
& \Rightarrow m^{\prime}(1 / n) \leqq m\left(1 / n^{2}\right)+2 / n \\
& \Rightarrow \lim _{n \rightarrow \infty} m^{\prime}(1 / n)=m^{\prime}(0)=m(0) \leqq \varliminf_{n \rightarrow \infty} m\left(1 / n^{2}\right)
\end{aligned}
$$

Recall that for all $n \geqq 1, m\left(1 / n^{2}\right) \leqq m(0)$. So $m(\cdot)$ is right continuous at zero.

Q.E.D.

Now we are ready to introduce our stronger version of Clarke's notion of calmness. Let $V=\left\{r: \boldsymbol{R}_{+} \rightarrow \overline{\boldsymbol{R}}_{+}=\boldsymbol{R}_{+} \cup\{+\infty\}\right.$ s.t. $r(\cdot)$ is nondecreasing and $\left.\lim _{\varepsilon \downarrow 0} r(\varepsilon)=r(0)=0\right\}$.

DEFInition. The problem of minimizing $J(\cdot)$ over the trajectories of $(*)_{1}$ (the "trajectory problem") is said to be "strongly calm" if and only if there 
exists $r(\cdot) \in V$ s.t. $\lim _{\varepsilon \downarrow 0} \frac{m(\varepsilon)-m(0)}{r(\varepsilon)}>-\infty$.

Remark. Clarke [6] defined calmness using $r(\varepsilon)=\varepsilon$.

Our next result shows that our notion of calmness is in fact equivalent to the well posedness of $(*)_{\varepsilon}$, in the sense of right continuity at zero of $m(\cdot)$.

THEOREM 4.2. If hypotheses $H(A), H(F)$ and $H(K)$ hold, them $m(\cdot)$ is right continuous at zero if and only if the trajectory problem is strongly calm.

Proof. $\Downarrow$ : Let $r(\varepsilon)=m(0)-m(\varepsilon) \geqq 0$. Since by hypothesis $m(\cdot)$ is right continuous at zero, we see that $r(\cdot) \in V$. Observe that $\frac{m(\varepsilon)-m(0)}{r(\varepsilon)}=-1$. So the trajectory problem is strongly calm.

$\Uparrow$ : Let $r(\cdot) \in V$ be the function postulated from the definition of strong calmness. We have:

$$
\begin{aligned}
& m(\varepsilon)-m(0)=\frac{m(\varepsilon)-m(0)}{r(\varepsilon)} \cdot r(\varepsilon) \\
& \Rightarrow \varliminf_{\varepsilon \downarrow 0}(m(\varepsilon)-m(0))=\varliminf_{\varepsilon \downarrow 0}\left[\frac{m(\varepsilon)-m(0)}{r(\varepsilon)} \cdot r(\varepsilon)\right] \\
& \geqq\left(\varliminf_{\varepsilon \downarrow 0} \frac{m(\varepsilon)-m(0)}{r(\varepsilon)}\right) \cdot\left(\lim _{\varepsilon \downarrow 0} r(\varepsilon)\right)=0
\end{aligned}
$$

since $\lim _{\varepsilon \downarrow 0} \frac{m(\varepsilon)-m(0)}{r(\varepsilon)}>-\infty$ (the problem being by hypothesis strongly calm). Hence we have $\lim _{\varepsilon \downarrow 0} m(\varepsilon) \geqq m(0)$.

On the other hand recall that $m(\varepsilon) \leqq m(0)$ for all $\varepsilon \geqq 0$. So $\varlimsup_{\varepsilon \downarrow 0} m(\varepsilon) \leqq m(0) \Rightarrow$ $\lim _{\varepsilon \downarrow 0} m(\varepsilon)=m(0) \Rightarrow m(\cdot)$ is right continuous at $0 . \quad{ }^{\varepsilon \downarrow 0} \quad$ Q.E.D.

Combining theorems 4.1 and 4.2 we get:

THEOREM 4.3. If hypotheses $H(A), H(F)$ and $H(K)$ hold, then $m^{\prime}(\cdot)$ is right continuous at zero if and only if the trajectry problem is strongly calm.

The next result will be useful in relating calmness and relaxability (i.e. $\left.m=m_{r}\right)$ of the trajectory problem. Let $r(\cdot) \in \varepsilon V$ and set $R(r)=\inf \{f(x)+k(x(0), x(b))$ $+r\left(\int_{0}^{b} d_{H}(x(t), K(t)) d t\right): \dot{x}(t)+A(t, x(t)) \in F(t, x(t)$ a.e. $\}$.

THEOREM 4.4. If hypotheses $H(A), H(F)$ and $H(K)$ hold, then $m(\cdot)$ is right continuous at zero if and only if there exist $r(\cdot) \in V$ s.t. $m(0)=R(r)$.

Proof. $\Downarrow$ : Let $x(\cdot) \in C(T, H)$ be a trajectory of $(*)_{1}$ (recall that we have assumed that $(*)_{1}$ admits trajectories). Note that 


$$
\begin{aligned}
& m\left(\int_{0}^{b} d_{H}(x(t), K(t)) d t\right) \leqq f(x)+k(x(0), x(b))=J(x) \\
& \Rightarrow m(0) \leqq J(x)+m(0)-m\left(\int_{0}^{b} d_{H}(x(t), K(t)) d t\right)
\end{aligned}
$$

Set $r(\varepsilon)=m(0)-m(\varepsilon)$. Due to the right continuity of $m(\cdot)$ at zero, $r(\cdot) \in V$. Since the last inequality above is true for any trajectory $x(\cdot)$ of $\dot{x}(t)+A(t, x(t))$ $\in F(t, x(t))$ a. e., we get that

$$
m(0) \leqq R(r)
$$

On the other hand, directly from the definitions we see that:

$$
\begin{aligned}
& R(r) \leqq m(0) \\
& \Rightarrow m(0)=R(r) .
\end{aligned}
$$

$\Uparrow$ : Given $\varepsilon>0, \delta>0$, let $x(\cdot)$ be a trajectory of $\dot{x}(t)+A(t, x(t)) \in F(t, x(t))$ a. e. s. t. $\int_{0}^{b} d(x(t), K(t)) d t \leqq \varepsilon$ and $J(x) \leqq m(\varepsilon)+\delta$. By hypothesis there exists $r(\cdot) \in V$ s.t.

$$
\begin{aligned}
& m(0)=R(r) \leqq J(x)+r\left(\int_{0}^{b} d_{H}(x(t), K(t)) d t\right) \leqq m(\varepsilon)+\delta+r(\varepsilon) \\
& \Rightarrow m(0) \leqq \varliminf_{\varepsilon \downarrow 0} m(\varepsilon)+\delta .
\end{aligned}
$$

Since $\delta>0$ was arbitrary, we deduce that:

$$
m(0) \leqq \underline{\lim } m(\varepsilon) .
$$

On the other hand, recall that we always have $m(\varepsilon) \leqq m(0), \varepsilon \geqq 0$. So

$$
\begin{aligned}
& \lim _{\varepsilon \downarrow 0} m(\varepsilon)=m(0) \\
& \Rightarrow m(\cdot) \text { is right continuous at zero. }
\end{aligned}
$$

Now we are ready for the main result of this section, which relates the concepts of well-posedness and relaxability (i. e. $\left.m=m_{r}\right)$. By $C\left(T, X_{w}\right)$ we will denote the space of weakly continuous $X$-valued functions defined on $T$.

THEOREM 4.5. If hypotheses $H(A)_{1}, H(F)_{1}, H(K)$ hold and $f: C\left(T, X_{w}\right) \rightarrow \boldsymbol{R}$ is continuous and bounded below by $\gamma_{1}$ and $k: X \times X \rightarrow \boldsymbol{R}$ continuous and bounded below by $\gamma_{2}$, then $m(\cdot)$ is right continuous at zero if and only if the trajectory problem is relaxable (i.e. $\left.m=m_{r}\right)$.

Proof. $\Downarrow$ : Suppose that this implication was false. Then $0<\delta=m-m_{r}$ and so we can find $x(\cdot)$ a trajectory of the relaxed system $(* *)_{1}$ s.t.

$$
J(x) \leqq m(0)-\delta / 2 .
$$


Since by hypothesis $\left({ }^{*}\right)_{1}$ has solutions and $J(\cdot)$ is continuous we can find an original trajectory (i. e. trajectory of $\left.\left({ }^{*}\right)_{1}\right), z(\cdot) \in C\left(T, X_{w}\right)$ s.t.

$$
|J(x)-J(z)| \leqq \delta / 6
$$

and since $X \hookrightarrow H$ compactly we can also have:

$$
r\left(\int_{0}^{b} d_{H}(z(t), K(t)) d t\right) \leqq \delta / 6 .
$$

where $r(\cdot) \in V$ and $R(r)=m(0)$ (see theorem 4.4).

Hence we have:

But then

$$
\begin{aligned}
J(z)+r\left(\int_{0}^{b} d_{H}(z(t), K(t)) d t\right) & \leqq J(x)+\delta / 6+\delta / 6=J(x)+\delta / 3 \\
& \leqq m(0)-\delta / 2+\delta / 3=m(0)-\delta / 6
\end{aligned}
$$

$$
m(0)=R(r) \leqq J(z)+r\left(\int_{0}^{b} d_{H}(z(t), K(t)) d t\right) \leqq m(0)-\delta / 6
$$

which is absurd. So the implication is true.

$\pi:$ Again we proceed by contradiction. So suppose that $\frac{\lim }{\varepsilon \downarrow 0} m(\varepsilon)<m(0)$. Let $\varepsilon_{n} \downarrow 0$ s.t. $m\left(\varepsilon_{n}\right) \rightarrow \varliminf_{\varepsilon \downarrow 0} m(\varepsilon)$. Set $\delta=m(0)-\frac{\lim }{\varepsilon \downarrow 0} m(\varepsilon)>0$. Then there exists $n_{0} \geqq 1$ s.t. for $n \geqq n_{0}$ we have:

$$
m\left(\varepsilon_{n}\right) \leqq m(0)-\delta / 2 .
$$

So we can find $x_{n}(\cdot) \in W(T) \subseteq C(T, H)$ satisfying $x_{n}(t)+A\left(t, x_{n}(t)\right) \in F\left(t, x_{n}(t)\right)$ a. e., $x_{n}(0) \in K(0)$ and $\int_{0}^{b} d_{H}\left(x_{n}(t), K(t)\right) d t \leqq \varepsilon_{n}$ s.t.

$$
\begin{aligned}
& J\left(x_{n}\right) \leqq m(0)-\delta / 3 \\
& \Rightarrow f\left(x_{n}\right)+k\left(x_{n}(0), x_{n}(b)\right) \leqq m(0)-\delta / 3 .
\end{aligned}
$$

Since $\left\{\overline{x_{n}(0)}\right\}_{n \geqq 1}^{w} \leqq K(0)$ is $w$-compact in $X$, as in the proof of theorem 3.1 we have that $K_{0}=\{y(\cdot) \in C(T, H): \dot{y}(t)+A(t, y(t))=h(t), y(0) \in C,\|h(t)\| \leqq a(t)$ a.e. $\}$ is sequentially compact in $C\left(T, X_{w}\right)$. Since $\left\{x_{n}\right\}_{n \geq 1} \subseteq K_{0}$, by passing to a subsequence if necessary, we may assume that $x_{n} \rightarrow x$ in $C\left(T, X_{w}\right)$. Also as in the proof of theorem 3.1, we can get that $\left\|\dot{x}_{n}\right\|_{q} \leqq M_{0} \Rightarrow\left\|\dot{x}_{n}\right\|_{1} \leqq M_{0}^{\prime}$ and thus from the Dunford-Pettis weak compactness criterion and by passing to an appropriate subsequence if necessary, we may assume that $\dot{x}_{n} \stackrel{w}{\rightarrow} z$ in $L^{1}\left(X^{*}\right)$. It is clear that $\dot{x}=z$. Using theorem 3.1 of [16], we have that:

$$
\begin{aligned}
\dot{x}(t) \in \overline{\operatorname{conv}} w-\overline{\lim }\left\{\dot{x}_{n}(t)\right\}_{n \geqq 1} \text { a. e. } & \\
\Rightarrow \dot{x}(t)+A(t, x(t)) & \in \overline{\operatorname{conv}} w-\overline{\lim }\left\{x_{n}(t)\right\}_{n \geqq 1}+w-\overline{\lim }\left\{A\left(t, x_{n}(t)\right)\right\}_{n \geqq 1} \\
& \leqq \overline{\operatorname{conv}} w-\overline{\lim } F\left(t, x_{n}(t)\right) \subseteq \overline{\operatorname{conv}} F(t, x(t)) \text { a. e. }
\end{aligned}
$$


Also $\int_{0}^{b} d_{H}\left(x_{n}(t), K(t)\right) d t \rightarrow 0$ as $n \rightarrow \infty$. Applying Fatou's lemma we get that:

$$
\begin{aligned}
& \int_{0}^{b} \underline{\lim } d_{H}\left(x_{n}(t), K(t)\right) d t \leqq \underline{\lim } \int_{0}^{b} d_{H}(x(t), K(t)) d t=0 \\
& \Rightarrow \int_{0}^{b} d_{H}(x(t), K(t)) d t=0 \\
& \Rightarrow d_{H}(x(t), K(t))=0 \text { a. e. } \\
& \Rightarrow x(\cdot) \text { is a relaxed viable trajectory. }
\end{aligned}
$$

Finally note that since relaxability holds, we have:

$$
m_{r}=m=m(0) \leqq J(x),
$$

and since $J\left(x_{n}\right) \rightarrow J(x)$ and $J\left(x_{n}\right) \leqq m(0)-\delta / 3$, we have

$$
\begin{aligned}
& J(x) \leqq m(0)-\delta / 3 \\
& \Rightarrow m(0) \leqq m(0)-\delta / 3
\end{aligned}
$$

which is absurd. So the implication holds.

Q.E.D.

Combining the results of this section we can have the following complete characterization of relaxability.

THEOREM 4.6. If the hypotheses of theorem 4.5 hold, then the following are equivalent:

(1) the trajectory problem is relaxable,

(2) $m(\cdot)$ is right continuous at zero,

(3) $m^{\prime}(\cdot)$ is right continuous at zero,

(4) the trajectory problem is strongly calm.

Remark. We could have defined strong calmness by requiring that $\varliminf_{\varepsilon \downarrow 0} \frac{m^{\prime}(\varepsilon)-m^{\prime}(0)}{r(\varepsilon)}>-\infty$ for some $r(\cdot) \in V$. The same results could have been obtained using this definition. However the definition using $m(\cdot)$ is more directly related to the notion of calmness of Clarke [6], which was defined through perturbations in the state constraints. By the way, note that Clarke [6], has only initial and terminal state constraints (i.e. for $t=0$ and $t=b$ ). Here the situation is more general in that respect.

\section{Relaxation of semilinear evolution inclusions.}

In this section, we concentrate our attention to a particular subclass of inclusions (*), namely the semilinear ones. This allows us to improve the hypotheses on the orientor field $F(t, x)$. 
So the multivalued Cauchy problem under consideration is :

$$
\left\{\begin{array}{l}
\dot{x}(t)+A(t) x(t) \in F(t, x(t)) \text { a. e. } \\
x(0)=x_{0} \in H
\end{array}\right\} \quad(*)_{2}
$$

with the following hypothese concerning the data of the problem:

$H(A)_{2}: \quad A: T \times X \rightarrow X^{*}$ is a map s.t.

(1) $t \rightarrow A(t) x$ is measurable,

(2) $x \rightarrow A(t) x$ is linear, monotone

(3) $\left\|A(t) x-A\left(t^{\prime}\right) x\right\|_{*} \leqq k\left|t-t^{\prime}\right| \cdot\|x\|$

(4) $\langle A(t) x, x\rangle \geqq c\|x\|^{2}$ a. e. $\left.c\right\rangle 0$ (i. e. $A(t)(\cdot)$ is a. e. strongly monotone),

(5) $\|A(t) x\| \leqq c^{\prime}\|x\| c^{\prime}>0$ (i. e. $A(t)(\cdot)$ is continuous).

For the orientor field $F(t, x)$, we will assume the following:

$H(F)_{2}: \quad F: T \times H \rightarrow P_{f c}(H)$ is a multifunction s.t.

(1) $(t, x) \rightarrow F(t, x)$ is graph measurable,

(2) $x \rightarrow F(t, x)$ is 1. s. c. from $H$ into $H$,

(3) $|F(t, x)|_{H}=\sup \{|y|: y \in F(t, x)\} \leqq a(t)+b(t)|x|$ a. e., with $a(\cdot), b(\cdot) \in L^{1}$.

Because of hypothesis $H(A)_{2}$ we know (see for example Tanabe [22], section 5.4), that the family $\{-A(t): t \in T\}$ of linear operators, generates a strongly continuous evolution operator $S: \Delta=\{(t, s): 0 \leqq s \leqq t \leqq b\} \rightarrow \mathcal{L}(H)$. We will make the following hypothesis concerning this operator.

$H_{c}: S(t, s)$ is compact for $t-s>0$.

THEOREM 5.1. If hypotheses $H(A)_{2}, H(F)_{2}$ and $H_{c}$ hold, then $(*)_{2}$ admits a solution belonging in $W(T) \subseteq C(T, H)$.

Proof. As in the proof of theorem 3.1, we will start by obtaining an a priori bound for the solutions of $(*)_{2}$. So let $x(\cdot)$ be such a solution. From proposition 5.5 .1 of Tanabe [21], we know that:

$$
\begin{aligned}
& x(t)=S(t, 0) x_{0}+\int_{0}^{t} S(t, s) f(s) d s, t \in T, f \in S_{F(\cdot, x(\cdot))}^{1} \\
& \Rightarrow|x(t)| \leqq M_{1}\left|x_{0}\right|+M_{1} \int_{0}^{t}(a(s)+b(s)|x(s)|) d s\left(\|S(t, s)\| \leqq M_{1}\right) \\
& \Rightarrow|x(t)| \leqq M_{1}\left(\left|x_{0}\right|+\|a\|_{1}+M_{1} \int_{0}^{t} b(s)|x(s)| d s .\right.
\end{aligned}
$$

Applying Gronwall's inequality, we get that:

$$
|x(t)| \leqq M_{2} \exp \left(M_{1} \cdot\|b\|_{1}\right)=\bar{M},
$$

where $M_{2}=M_{1}\left(\left|x_{0}\right|+\|a\|_{1}\right)$. 
Now consider the multifunction $\hat{F}: T \times H \rightarrow P_{f}(H)$ defined by :

$$
\hat{F}(t, x)= \begin{cases}F(t, x) & \text { if }|x| \leqq \bar{M} \\ F\left(t, \frac{\bar{M} x}{|x|}\right) & \text { if }|x|>\bar{M} .\end{cases}
$$

Clearly then $\hat{F}(t, x)=F\left(t, p_{\bar{M}}(x)\right)$, where $p_{\bar{M}}(\cdot)$ is the $\bar{M}$-radial retraction in $H$. Recall that $p_{\bar{M}}(\cdot)$ is Lipschitz continuous. So $x \rightarrow \hat{F}(t, x)$ is 1. s.c.. We will also show that $(t, x) \rightarrow \hat{F}(t, x)$ is graph measurable. Let $\eta: T \times H \times H \rightarrow T \times H \times H$ be defined by $\eta(t, x, z)=\left(t, p_{\bar{M}}(x), z\right)$. Clearly this map is $B(T) \times B(H) \times B(H)$ measurable. Now note that:

$$
\begin{aligned}
G r \hat{F} & =\left\{(t, x, z) \in T \times H \times H: z \in \hat{F}(t, x)=F\left(t, p_{\bar{M}}(x)\right)\right\} \\
& =\{(t, x, z) \in T \times H \times H: \eta(t, x, z) \in G r F\} .
\end{aligned}
$$

But by hypothesis $G r F \in B(T) \times B(H) \times B(H)$ and we saw that $\eta(\cdot, \cdot, \cdot)$ is measurable. Hence $G r \hat{F} \in B(T) \times B(H) \times B(H)$ i. e. $\hat{F}(\cdot, \cdot)$ is graph measurable. Also we will show that if $x: T \rightarrow H$ is measurable, then $S_{\hat{f}(\cdot, x(\cdot))}^{1} \neq \varnothing$. Now let $h: T \times H \rightarrow T \times H \times H$ be defined by $h(t, z)=(t, x(t), z)$. Clearly this map is $(B(T) \times B(H), B(T) \times B(H) \times B(H))$-measurable. Then we have:

$$
G r \hat{F}(\cdot, x(\cdot))=\{(t, z) \in T \times X: h(t, z) \in G r \hat{F}\} .
$$

Since $h(\cdot, \cdot)$ is measurable and $\operatorname{Gr} \hat{F} \in B(T) \times B(H) \times B(H)$, we deduce that $\operatorname{Gr} \hat{F}(\cdot, x(\cdot)) \in B(T) \times B(H) \Rightarrow t \rightarrow \hat{F}(t, x(t))$ is $\hat{B}(T)$-measurable, where $\hat{B}(T)$ is the Lebesgue completion of the Borel $\sigma$-field $B(T)$. Finally note that:

$$
|\hat{F}(t, x)|_{H} \leqq \phi(t)=a(t)+\bar{M} b(t) \text { a. e., } \phi(\cdot) \in L_{+}^{1} \text {, so } S_{\hat{F}(\cdot, x(\cdot))}^{1} \neq \varnothing .
$$

Having established all these properties about $\hat{F}(\cdot, \cdot)$, we now proceed as follows. Consider $V \cong L^{1}(H)$ defined by :

$$
V=\left\{g \in L^{1}(H):|g(t)| \leqq \phi(t) \text { a.e. }\right\}
$$

Since $H$ is a Hilbert space, through the Dunford-Pettis compactness criterion, it is easy to check that $V$ is sequentially weakly compact in $L^{1}(H)$. Let $g(\cdot) \in V$ and consider the following single valued evolution equation:

$$
\left\{\begin{array}{l}
\dot{x}(t)+A(t) x(t)=g(t) \text { a.e. } \\
x(0)=x_{0}
\end{array}\right\} \quad(*)_{2}(g)
$$

From Barbu [2] (p. 166), we know that $(*)_{2}(g)$ has a unique solution $u(g)(\cdot) \in W(T)$. Let $K=\{u(g)(\cdot) \in W(T) \subseteq C(T, H): g \in V\}$. We claim that $K$ is compact in $C(T, H)$. To this end, let $x(\cdot) \in K, t, t^{\prime} \in T, t<t^{\prime}$. We have: 


$$
\begin{aligned}
& \left|x\left(t^{\prime}\right)-x(t)\right| \leqq\left|S\left(t^{\prime}, 0\right) x_{0}-S(t, 0) x_{0}\right|+\left|\int_{0}^{t^{\prime}} S\left(t^{\prime}, s\right) g(s) d s-\int_{0}^{t} S(t, s) g(s) d s\right| \\
& \leqq\left|S\left(t^{\prime}, 0\right) x_{0}-S(t, 0) x_{0}\right|+\int_{t}^{t^{\prime}}\left\|S\left(t^{\prime}, s\right)\right\| \cdot|g(s)| d s+\int_{0}^{t}\left\|S\left(t^{\prime}, s\right)-S(t, s)\right\| \cdot|g(s)| d s \\
& \leqq\left|S\left(t^{\prime}, 0\right) x_{0}-S(t, 0) x_{0}\right|+M_{1} \int_{t}^{t^{\prime}} \phi(s) d s+\int_{0}^{t}\left\|S\left(t^{\prime}, s\right)-S(t, s)\right\| \cdot \phi(s) d s .
\end{aligned}
$$

Because of the strong continuity of the evolution operator $S(\cdot, \cdot)$, given $\varepsilon>0$, there exists $\delta_{1}>0$ s.t. for $\left|t^{\prime}-t\right|<\delta_{1}$ we have $\left|S\left(t^{\prime}, 0\right) x_{0}-S(t, 0) x_{0}\right|<\varepsilon / 3$. Also let $\delta_{2}>0$ s.t. if $\left|t^{\prime}-t\right|<\delta_{2}$, then $M_{1} \int_{t}^{t^{\prime}} \phi(s) d s<\varepsilon / 3$. Finally since $S(t, s)$ is compact for $t-s>0$ (hypothesis $H_{c}$ ), from proposition 2.1 of [15], we know that $t \rightarrow S(t, s)$ is continuous in the operator norm, uniformly in $s$ for $t-s$ bounded away from zero. So let $\varepsilon^{\prime}>0$ and $\delta_{3}>0$ be such that if $\left|t^{\prime}-t\right|<\delta_{3}$, then

$$
\int_{t-\varepsilon^{\prime}}^{t}\left\|S\left(t^{\prime}, s\right)-S(t, s)\right\| \cdot \phi(s) d s \leqq 2 M_{1} \int_{t-\varepsilon^{\prime}}^{t} \phi(s) d s<\varepsilon / 6
$$

and

$$
\int_{0}^{t-\varepsilon^{\prime}}\left\|S\left(t^{\prime}, s\right)-S(t, s)\right\| \cdot \phi(s) d s<\varepsilon / 6
$$

Combining all the above estimates, we get that for $\left|t^{\prime}-t\right|<\delta=\min \left(\delta_{1}, \delta_{2}, \delta_{3}\right)$ and for all $x(\cdot) \in K$, we have:

$$
\begin{aligned}
& \left|x\left(t^{\prime}\right)-x(t)\right|<\varepsilon \\
& \Rightarrow K \subseteq C\left(T, H^{\prime}\right) \text { is equicontinuous. }
\end{aligned}
$$

Also since $S(t, s)$ is compact for $t-s>0$, using the Rädstrom embedding theorem (see for example Hiai-Umegaki [10]), we have that:

$$
\left\{\int_{0}^{t} S(t, s) g(s) d s: g \in V\right\} \in P_{k}(H) \Rightarrow c l\{x(t): x(\cdot) \in K\} \in P_{k}(H), t \in T .
$$

Finally we will show that $K$ is closed in $C(T, H)$. To this end let $x_{n} \rightarrow x$ in $C(T, H), x_{n}(\cdot) \in K$. We have :

$$
x_{n}(t)=S(t, 0) x_{0}+\int_{0}^{t} S(t, s) g_{n}(s) d s, g_{n}(\cdot) \in V .
$$

By passing to a subsequence if necessary, we may assume that $g_{n} \stackrel{w}{\rightarrow} g \in V$ in $L^{1}(H)$. Then $\int_{0}^{t} S(t, s) g_{n}(s) d s \stackrel{w}{\rightarrow} \int S(t, s) g(s) d s$ in $H$. So in the limit we have:

$$
\begin{aligned}
& x(t)=S(t, 0) x_{0}+\int_{0}^{t} S(t, s) g(s) d s \\
& \Rightarrow x=u(g) \\
& \Rightarrow x \in K \text { and so } K \text { is closed in } C(T, H) .
\end{aligned}
$$


Therefore, invoking the Arzela-Ascoli theorem, we conclude that $K$ is compact in $C(T, H)$. Then by Mazur's theorem $\hat{K}=\overline{\text { conv }} K$ is compact in $C(T, H)$.

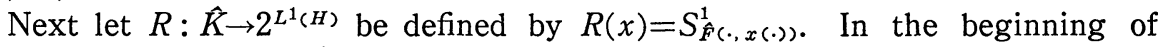
the proof we saw that $\hat{F}(\cdot, x(\cdot))$ is measurable and $L^{1}$-bounded by $\phi(\cdot)$. Hence $R(x) \neq \varnothing$ for all $x \in \hat{K}$. In fact $R(x) \in P_{f}\left(L^{1}(H)\right)$. Also using hypothesis $H(F)_{2}$ (2) and theorem 4.1 of [16], we get that $R(\cdot)$ is a 1.s.c. multifunction. Apply Fryszkowski's selection theorem [9], to get $r: \hat{K} \rightarrow L^{1}(H)$ continuous s.t. $r(x) \in R(x)$ for all $x \in \hat{K}$.

Now consider the following evolution equation:

$$
\left\{\begin{array}{l}
\dot{x}(t)+A(\dot{t}) x(t)=r(y)(t) \text { a.e. } \\
x(0)=x_{0}
\end{array}\right\} \quad(*)_{2}(y)
$$

where $y \in \hat{K}$. Let $x(y)(\cdot)=q(y)(\cdot)$ be the unique solution of $(*)_{2}(y)$. Clearly $q: \hat{K} \rightarrow \hat{K}$ and it is easy to check that it is continuous. Apply Schauder's fixed point theorem to find $\hat{x} \in \hat{K}$ s.t. $\hat{x}=q(\hat{x})$. Clearly $\hat{x}(\cdot)$ solves $(*)_{2}$ for the orientor field $\hat{F}(t, x)$. As in the beginning of the proof, through Gronwall's inequality, we get that $|\hat{x}(t)| \leqq \bar{M} \Rightarrow F(t, \hat{x}(t))=\hat{F}(t, \hat{x}(t)) \Rightarrow \hat{x}(\cdot) \in W(T) \leqq C(T, H)$ is the desired solution of $(*)_{2}$.

Q.E.D.

This leads to the following relaxation result for $(*)_{2}$.

So we introduce the relaxed inclusion:

$$
\left\{\begin{array}{l}
\dot{x}(t)+A(t) x(t) \in \overline{\operatorname{conv}} F(t, x(t)) \text { a. e. } \\
x(0)=x_{0}
\end{array}\right\} \quad(* *)_{2} .
$$

Denote the solution set of $(*)_{2}$ by $Q\left(x_{0}\right)$ and the solution set of $\left(*^{* *}\right)_{2}$ by $Q_{r}\left(x_{0}\right)$.

THEOREM 5.2. If hypotheses $H(A)_{2}, H(F)_{2}$ and $H_{c}$ hold, then $\varnothing \neq \overline{Q\left(x_{0}\right)}=$ $Q_{r}\left(x_{0}\right)$, the closure taken in $C(T, H)$.

Proof. Let $y \in Q_{r}\left(x_{0}\right)$ and let $\varepsilon>0$. Pick $\delta>0$ s.t. for $A \subseteq T$ Lebesque measurable with $\lambda(A)<\delta$, we have:

$$
\int_{A}|F(s, y(s))|_{H} d s<\varepsilon / 4 M_{1}
$$

where $|S(t, s)| \leqq M_{1}$ for all $0 \leqq s \leqq t \leqq b$. Let $0=t_{0}<t_{1}<\cdots<t_{n}=b$ be an equipartition of $T=[0, b]$ s.t. $t_{k}-t_{k-1}<\delta$ for $k \in\{1,2, \cdots, n\}$. By definition we have:

$$
\begin{aligned}
& y(t)_{1} \in S\left(t_{1}, 0\right) x_{0}+\int_{0}^{t_{1}} S\left(t_{1}, s\right) \overline{\operatorname{conv}} F(s, y(s)) d s \\
& \Rightarrow y\left(t_{1}\right)=S\left(t_{1}, 0\right) x_{0}+q_{1}\left(t_{1}\right)
\end{aligned}
$$


where $q_{1}\left(t_{1}\right) \in \int_{0}^{t_{1}} S\left(t_{1}, s\right) \overline{\operatorname{conv}} F(s, y(s)) d s$. From theorem 4.2 of [13], we know that for $0<\delta^{\prime} \leqq\left(\varepsilon^{\prime} / \sum_{k-1}^{n} M_{1}^{k-1}\right)\left(\varepsilon^{\prime}=\min \left(\varepsilon / 2, \varepsilon / 2 M_{1}\right)\right)$, we can find $f_{1} \in S_{F^{\prime}(\cdot, y(\cdot))}^{1}$ s.t.

$$
\left|q_{1}\left(t_{1}\right)-\int_{0}^{t_{1}} S\left(t_{1}, s\right) f_{1}(s) d s\right|<\delta^{\prime}
$$

For $t \in\left[0, t_{1}\right]$, set $x_{1}(t)=S(t, 0) x_{0}+\int_{0}^{t} S(t, s) f_{1}(s) d s$. Then clearly we have:

$$
\left|x_{1}\left(t_{1}\right)-y\left(t_{1}\right)\right|<\delta^{\prime}
$$

Next we pass to the second subinterval $\left[t_{1}, t_{2}\right]$. Again we have:

$$
y\left(t_{2}\right)=S\left(t_{2}, t_{1}\right) y\left(t_{1}\right)+q_{2}\left(t_{2}\right)
$$

where $q_{2}\left(t_{2}\right) \in \int_{t_{1}}^{t_{2}} S\left(t_{2}, s\right) \overline{\operatorname{conv}} F(s, y(s)) d s$. Let $f_{2} \in S_{F(\cdot, y(\cdot))}^{1}$ be s.t.

$$
\left|q_{2}\left(t_{2}\right)-\int_{t_{1}}^{t_{2}} S\left(t_{2}, s\right) f_{2}(s) d s\right|<\delta^{\prime}
$$

Set $x_{2}(t)=S\left(t, t_{1}\right) x_{1}\left(t_{1}\right)+\int_{t_{1}}^{t} S(t, s) f_{2}(s) d s$, for $t \in\left[t_{1}, t_{2}\right]$. Then we have:

$$
\begin{aligned}
\left|x_{2}\left(t_{2}\right)-y\left(t_{2}\right)\right| & =\left|S\left(t_{2}, t_{1}\right) x_{1}\left(t_{1}\right)+\int_{t_{1}}^{t_{2}} S\left(t_{2}, s\right) f_{2}(s) d s-S\left(t_{2}, t_{1}\right) y\left(t_{1}\right)-q_{2}\left(t_{2}\right)\right| \\
& \leqq M_{1} \delta^{\prime}+\delta^{\prime}=\delta^{\prime}\left(M_{1}+1\right) .
\end{aligned}
$$

Continuing this way, we get $f_{k} \in S_{F(\cdot, y(\cdot))}^{1}$ and define for $t \in\left[t_{k-1}, t_{k}\right]$, $k \in\{1,2, \cdots, n\}, \quad x_{k}(t)=S\left(t, t_{k-1}\right) x_{k-1}\left(t_{k-1}\right)+\int_{t_{k-1}}^{t} S(t, s) f_{k}(s) d s, \quad$ for which we have:

$$
\left|x_{k}\left(t_{k}\right)-y\left(t_{k}\right)\right|<\delta^{\prime} \sum_{r=1}^{k} M_{1}^{r-1}
$$

If we set $\hat{f}=\sum_{k=1}^{n} \chi_{\left[t_{k-1}, t_{k}\right]}(\cdot) f_{k}(\cdot)$, we see that $\hat{f} \in S_{F(\cdot, y(\cdot))}^{1}$ and $x(t)=S(t, 0) x_{0}$ $+\int_{0}^{t} S(t, s) \hat{f}(s) d s$ is a solution of $(*)_{2}$. Furthermore from our construction we have $\left|x\left(t_{k}\right)-y\left(t_{k}\right)\right|<\varepsilon^{\prime}$ for all $k \in\{1,2, \cdots, n\} \quad$ (recall $\delta^{\prime} \leqq\left(\varepsilon^{\prime} / \sum_{k=1}^{n} M_{1}^{k-1}\right)$ ).

Next let $t \in T$ be arbitrary. Then $t \in\left[t_{k}, t_{k+1}\right]$ for some $k \in\{0,1, \cdots, n-1\}$. So if $g \in S^{1} \overline{\text { conv } F(\cdot, y(\cdot))}$ generates the relaxed trajectory $y(\cdot)$, we have

$$
\begin{aligned}
& |x(t)-y(t)| \leqq \min M_{1}\left(\varepsilon / 2, \varepsilon / 2 M_{1}\right)+\varepsilon / 2 \\
& \Rightarrow\|x-y\|_{C(T, H)}<\varepsilon \\
& \Rightarrow \overline{Q\left(x_{0}\right)}=Q_{r}\left(x_{0}\right) \text { the closure in } C(T, H) .
\end{aligned}
$$

Q.E.D. 


\section{Relaxability and calmness of semilinear systems.}

As before we introduce a cost criterion of the following form:

$$
J(x)=f(x)+k(x(0), x(b))
$$

where $f: C(T, H) \rightarrow \boldsymbol{R}$ and $k: H \times H \rightarrow \boldsymbol{R}$ are both continuous. Again we introduce a state constraint in the form of a time varying set $K(t)$. For this multifunction we will make the following hypothesis:

$H(K)_{1}: \quad K: T \rightarrow P_{f}(H)$ is measurable and $K(0) \subseteq H$ is compact.

As in the nonlinear case, knowing the a priori bounds for the trajectories of $(*)_{2}$, there is no loss of generality in assuming that $K(\cdot)$ is integrably bounded.

We will consider the following variational problem ("trajectory problem" with state constraints):

$$
\begin{gathered}
\inf \{f(x)+k(x,(0), x(b)): x(\cdot) \in C(T, H), x(t) \\
+A(t) x(t) \in F(t, x(t)) \text { a. e., } x(t) \in K(t)\} .
\end{gathered}
$$

We will assume that there exist feasible (viable) trajectories and that the value of the problem is finite. Note that since $K(0) \in P_{k}(H)$, the set of feasible trajectories is compact in $C(T, H)$ and the infimum is attained (hence the value of the problem is finite).

As in the nonlinear case we introduce two new problems. One with the state constraints perturbed and the other with the state constraints absorbed in the cost functional (penalized problem). So we have:

$$
m(a)=\inf \left\{J(x): \dot{x}(t)+A(t) x(t) \in F(t, x(t)) \text { a.e. } \int_{0}^{b} d_{H}(x,(t), K(t)) d t \leqq a\right\}
$$

and $\quad m^{\prime}(a)=\inf \left\{J(x)+1 / a \int_{0}^{b} d_{H}(x(t)), K(t)\right) d t: x(t)+A(t) x(t) \in F(t, x(t))$ a.e. $\}$.

Again $m(0)=m^{\prime}(0)=m=$ the value of the minimization of $J(\cdot)$ over the trajectories of $\dot{x}(t)+A(t) x(t) \in F(t, x(t))$ a. e., $x(t) \in K(t)$. Similarly $m_{r}$ will be the value of the minimization of $J(\cdot)$ over the trajectories of $\dot{x}(t)+A(t) x(t)$ $\in \overline{\mathrm{conv}} F(t, x(t))$ a. e., $x(t) \in K(t)$ ("relaxed variational problem"). We will say that the state constrained system is "relaxable" if and only if $m=m_{r}$.

As for nonlinear systems (see section 4), we have the following complete characterization of relaxability:

THEOREM 6.1. If $H(A)_{2}, H(F)_{2}, H(K)_{1}$ hold and $f: C(T, H) \rightarrow \boldsymbol{R}, k: H \times H \rightarrow \boldsymbol{R}$ are continuous and bounded below, then the following are equivalent.

(1) the trajectory problem is relaxable,

(2) $m(\cdot)$ is right continuous at zero,

(3) $m^{\prime}(\cdot)$ is right continuous at zero,

(4) the trajectry problem is strongly calm. 


\section{Control problems.}

To see that the trajectory problem studied in the previous sections, subsumes the optimal control problem, consider the following nonlinear, infinite dimensional control system, with time varying control constraints:

$$
\left\{\begin{array}{l}
\dot{x}(t)+A(t) x(t)=f(t, x(t), u(t)) \text { a. e. } \\
x(0)=x_{0} \in H, u(t) \in U(t) \text { a. e., } u(\cdot)=\text { measurable }
\end{array}\right\}
$$

Here $T, X, H, X^{*}$ are as before, while $Y$ is a separable Banach space, modelling the control space.

We will need the following hypotheses concerning $f(\cdot, \cdot, \cdot)$ and $U(\cdot)$.

$H(f): F: T \times H \times Y \rightarrow H$ is a map s.t.

(1) $t \rightarrow f(t, x, y)$ is measurable,

(2) $x \rightarrow f(t, x, y)$ is continuous from $H$ into $H$,

(3) $y \rightarrow f(t, x, y)$ is continuous from $Y_{w}$ into $H_{w}^{\prime}$,

(4) $|f(t, x, y)| \leqq a(t)+b(t)|x|$ a. e., with $a(\cdot), b(\cdot) \in L_{+}^{1}$.

$H(U): U: T \rightarrow P_{f c}(Y)$ is measurable and $U(t) \cong K$ a. e. with $K \in P_{w k c}(Y)$.

Set $F(t, x)=\bigcup_{u \in U(t)} f(t, x, u)$ and consider the following evolution inclusions:

$$
\left\{\begin{array}{l}
\dot{x}(t)+A(t) x(t) \in F(t, x(t)) \text { a. e. } \\
x(0)=x_{0} \in H
\end{array}\right\} \quad(* * *)_{1}
$$

Proposition 7.1. If $H(A)_{2}, H(f)$ and $H(U)$ hold, then $(* * *)$ and $(* * *)_{1}$ are equivalent systems.

Proof. Let $x(\cdot)$ be a trajectory of $(* * *)$ corresponding to the control function $u(\cdot)$. Since $f(t, x(t), u(t)) \in F(t, x(t))$, we only need to show that $t \rightarrow f(t, x(t), u(t))$ is measurable from $T$ into $H$. To see this fix $x \in H$ and for $h \in H$, consider the map $(t, u) \rightarrow(h, f(t, x, u))$. This map is measurable in $t$, continuous in $u$ (i. e. is a Caratheodory map), hence is jointly measurable. Then, $(t, x, u) \rightarrow(h, f(t, x, u))$ is Caratheodory too (measurable in $(t, u)$, continuous in $x)$. Thus it is jointly measurable. So $t \rightarrow(h, f(t, x(t), u(t))$ is measurable and since $h \in H$ was arbitrary, $t \rightarrow f(t, x(t), u(t))$ is Pettis (weakly) measurable. But recall that $H$ is separable. So from the Pettis measurability theorem, we deduce that $t \rightarrow f(t, x(t), u(t))$ is strongly measurable $\Rightarrow f(\cdot, x(\cdot), u(\cdot)) \in S_{F^{\prime}(\cdot . x(\cdot))}^{1} \Rightarrow x(\cdot)$ solves $(* * *)_{1}$.

Next let $x(\cdot)$ be a solution of $(* * *)_{1}$. Then we have:

$$
x(t)=S(t, 0) x_{0}+\int_{0}^{t} S(t, s) g(s) d s, t \in T, g \in S_{F(\cdot, x(\cdot))}^{1} .
$$

Let $R(t)=\{u \in U(t): g(t)=f(t, x(t), u)\}$. Take $\left\{h_{n}\right\}_{n \geqq 1}$ be dense in $H$. Recall that for each $n \geqq 1$, the $\operatorname{map}(t, u) \rightarrow k_{n}(t, u)=\left(h_{n}, g(t)-f(t, x(t), u)\right)$ is measurable. 
Then rewrite $R(\cdot)$ as follows:

$$
\begin{aligned}
& R(t)=\bigcap_{n \geq 1}\left\{u \in U(t): k_{n}(t, u)=0\right\} \\
& \Rightarrow G r R=\bigcap_{n \geqq 1}\left\{(t, u) \in G r U: k_{n}(t, u)=0\right\} \in B(T) \times B(Y),
\end{aligned}
$$

since $G r U \in B(T) \times B(Y)(U(\cdot)$ being by hypothesis measurable). Apply Aumann's selection theorem (see Saint-Beuve [19], theorem 3), to get $u: T \rightarrow Y$ measurable s.t. $u(t) \in U(t)$ a. e. $\Rightarrow g(t)=f(t, x(t), u(t))$ a. e., $u \in S_{U}^{1} \Rightarrow x(\cdot)$ solves $(* * *)$.

Remark. A careful look on the proof, can convince the reader that the hypotheses of the above proposition can be weakened considerably, without affecting the conclusion. However, since we will need them in the next, main result of this section, for the sake of uniformity in the exposition, we introduced them from the beginning.

THEOREM 7.1. If $H(A)_{2}, H(f)$ and $H(U)$ hold, then $F(t, x)$ satisfies $H(F)_{2}$ and so the contool problem is a special case of the trajectory problem.

Proof. First we will show that $F(\cdot, \cdot)$ has closed values. Let $z_{n} \stackrel{s}{\rightarrow} z$, $z_{n} \in F(t, x)$. Then by definition $z_{n}=f\left(t, x, u_{n}\right), u_{n} \in U(t) \subseteq K$. By passing to a subsequence if necessary, we may assume that $u_{n} \stackrel{w}{\rightarrow} u \in U(t)$. Then because of $H(f)(3), f\left(t, x, u_{n}\right) \stackrel{w}{\rightarrow} f(t, x, u) \Rightarrow z=f(t, x, u) \Rightarrow z \in F(t, x) \Rightarrow F(t, x) \in P_{f}(H)$.

Next we will show that $F(\cdot, \cdot)$ satisfies $H(F)_{2}(1)$. By definition we have:

$$
\begin{aligned}
G r F & =\{(t, x, z) \in T \times H \times H: z \in F(t, x)\} \\
& =\{(t, x, z) \in T \times H \times H: z=f(t, x, u), u \in U(t)\} .
\end{aligned}
$$

Let $\lambda(t, x, z, u)=z-f(t, x, u), \mu(t, u)=d_{Y}(u, U(t))$. Then

$$
G r F=\operatorname{proj}_{T \times H \times H}\{(t, x, z, u) \in T \times H \times H \times K: \lambda(t, x, z, u)=0, \mu(t, u)=0\} .
$$

Since $\lambda$ and $\mu$ are both measurable and $K \in P_{w k c}(Y)$, then as in the proof of theorem 3.2, through the Arsenin-Novikov theorem (see Saint-Beuve [21]), we get that $G r F \in B(T) \times B(H) \times B(H)$.

Next we will show that $H(F)_{2}(2)$ holds. We need to show that given $C \subseteq H$ nonempty, closed we have that $F^{+}(t, C)=\{x \in H: F(t, x) \subseteq C\}$ is closed too. Let $z_{n} \in F^{+}(t, C)$ s.t. $z_{n} \stackrel{s}{\rightarrow} z$ in $H$. Then $F\left(t, z_{n}\right) \subseteq C \Rightarrow f\left(t, z_{n}, u\right) \subseteq C$ for all $u \in U(t)$. Because of $H(f)(2)$, in the limit we get that $f(t, z, u) \in C$ for all $u \in U(t) \Rightarrow$ $F(t, z) \subseteq C \Rightarrow z \in F^{+}(t, C)$.

Finally note that $|F(t, x)|_{H}=|f(t, x, U(t))|_{H} \leqq a(t)+b(t)|x|$ a.e., with $a(\cdot)$, $b(\cdot) \in L_{+}^{1}$. So $H(F)(4)$ is satisfied. Hence $F(t, x)$ satisfies $H(F)$ and so the control problem is a special case of the trajectory problem studied in the previous sections.

Q.E.D. 
Having this theorem and assuming that $H_{c}$ holds, we can conclude that the given control system is relaxable.

\section{An example.}

We will illustrate the applicability of our work, with an example of a distributed parameter control system with state constraints.

So let $T=[0, b]$ and $W$ a bounded domain in $\boldsymbol{R}^{n}$ with smooth boundary $\Gamma=\partial W$. We will consider a nonlinear control system governed by the following nonlinear, parabolic partial differential equation defined on $T \times W$ :

$$
\left\{\begin{array}{l}
\frac{\partial x(t, z)}{\partial z}-\Delta x(t, z)=f(t, z, x(t, z)) u(t, z) \\
x(t, z)=0 \text { on } T \times \Gamma \\
u(\cdot, \cdot) \in M \\
g(t, z, x(t, z)) \leqq 0 \text { a.e. on } T \times W \\
|x(0, z)| \leqq \theta(z) \text { a.e. } \theta(\cdot) \in L_{+}^{2}
\end{array}\right\}
$$

We are also given the following integral cost functional:

$$
\int_{0}^{b} \int_{W} h(t, z, x(t, z), u(t, z)) d z d t .
$$

We have to minimize the above integral functional over all admissible "state-control" pairs of system (****).

We will make the following hypotheses:

A : $f: T \times W \times \boldsymbol{R} \rightarrow \boldsymbol{R}^{m}$

(1) $(t, z) \rightarrow f(t, z, r)$ is measurable,

(2) $r \rightarrow f(t, z, r)$ is continuous,

(3) $|f(t, z, r) u| \leqq a(t, z)+b(t, z)|r|$ a. e. for all $u \in \boldsymbol{R}^{m}$, with $a \in L^{2}(T \times W)$ $b(\cdot, \cdot)$ jointly measurable, $b(t, \cdot) \in L^{\infty}(W)$ and $t \rightarrow\|b(t, \cdot)\|_{L^{\infty}(W)}$ is an $L_{+}^{2}$-function.

B : $M$ is a weakly compact subset of $L^{2}(T \times W)$.

C: $g: T \times W \times \boldsymbol{R} \rightarrow \boldsymbol{R}$ is a function s.t.

(1) $(t, z) \rightarrow g(t, z, r)$ is measurable,

(2) $r \rightarrow g(t, z, r)$ is continuous,

(3) for every $t \in T$ and every $x(\cdot) \in L^{2}(W), g(t, \cdot, x(\cdot)) \in L^{2}(W)$

D: $h: T \times W \times \boldsymbol{R} \times \boldsymbol{R}^{m} \rightarrow \boldsymbol{R}$ is a function s.t.

(1) $(t, z, r, u) \rightarrow h(t, z, r, u)$ is measurable,

(2) $(r, u) \rightarrow h(t, z, r, u)$ is 1 . s. c. and convex in $u$,

(3) there exists $\lambda \in L^{2}(T \times W)$ s.t. for all $(x, u) \in \boldsymbol{R} \times \boldsymbol{R}^{m}, \lambda(t, z) \leqq h(t, z, x, u)$ a.e.

$\mathrm{E}:$ System $(* * * *)$ has solutions.

Let $X=H_{0}^{1}(W), H=L^{2}(W)$ and $Y=L^{2}\left(W, \boldsymbol{R}^{m}\right)$. The operator $A: X \rightarrow X^{*}$ is 
defined by the Dirichlet form $\alpha(\phi, \phi)=\int_{W} \nabla \phi(z) \cdot \nabla \phi(z) d z$, for all $\phi, \phi \in X$. So $\alpha(\phi, \phi)=\langle A \phi, \phi\rangle$. So it is easy to check that it satisfies $H(A)_{2}$. Also it generates a linear contractions semigroup $S(t): L^{2}(W) \rightarrow L^{2}(W)$, which from theorem 5.2 of Pavel [18], we know that it is compact for $t>0$. So hypothesis $H_{c}$ is satisfied.

Next let $w: T \times H \times Y \rightarrow H$ be defined by $w(t, x, u)=f(t, \cdot, x(\cdot)) u(\cdot)$. Hypothesis $A(3)$ and Krasnoselski's theorem, tell us that $t \rightarrow w(t, x, u)$ is measurable, while $x \rightarrow w(t, x, u)$ is continuous. Furthermore $w(t, x, \cdot)$ is linear, hence $u \rightarrow$ $w(t, x, u)$ is weakly continuous. Finally $|w(t, x, u)|_{H=L^{2}(w)} \leqq \hat{a}(t)+b(t)|x|_{H}$ a. e.,

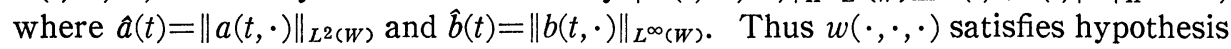
$H(f)$.

Note that in this example the control constraint set is globally and not pointwise defined.

Next let $\hat{g}: T \times H \rightarrow H$ be defined by :

$$
\hat{g}(t, x)(\cdot)=g(t, \cdot, x(\cdot))
$$

Clearly then $(t, x) \rightarrow \hat{g}(t, x)$ is measurable. Set

$$
K(t)=\{x \in H: \hat{g}(t, x)(z) \leqq 0 \text { a. e. }\}=\{x \in H: g(t, z, x(z)) \leqq 0 \text { a. e. }\} \text {. }
$$

It is clear that $K(\cdot)$ is closed valued, and since $(t, x) \rightarrow \hat{g}(t, x)$ is measurable, $K(\cdot)$ is a measurable multifunction. Thus it satisfies hypothesis $H(K)_{i}$.

Now rewrite system $(* * * *)$, as the following evolution equation:

$$
\left\{\begin{array}{l}
\dot{x}(t)+A x(t)=w(t, x(t), u(t)) \text { a. e. } \\
x(t) \in K(t), u \in M
\end{array}\right\} \quad(* * * *)_{1}
$$

Also let $\hat{h}: T \times H \times Y \rightarrow \boldsymbol{R}$ be defined by:

$$
\hat{h}(t, x, u)=\int_{W} h(t, z, x(z), u(z)) d z
$$

From hypothesis $(D)$ and theorem 2.1, p. 243 of Ekeland-Teman [7] we see that $\hat{h}(t, \cdot, \cdot)$ is 1 . s. c. on $H \times Y_{w}$, while clearly $h(\cdot, \cdot, \cdot)$ is jointly measurable. Rewrite the cost functional as follows:

$$
J(\hat{x}, \hat{u})=\int_{0}^{b} \hat{h}(t, \hat{x}(t), \hat{u}(t)) d t
$$

where $\hat{x}(t)(\cdot)=x(t, \cdot)$ and $\hat{u}(t)=u(t, \cdot)$.

Our claim is that for this optimal control problem, the value function $m(\cdot)$ is right continuous at zero. So let $\varepsilon_{n} \downarrow 0$ and let $\left(x_{n}, u_{n}\right)$ be admissible pairs for the corresponding perturbed optimal control problems s.t.

$$
J\left(x_{n}, u_{n}\right) \leqq m\left(\varepsilon_{n}\right)+1 / n .
$$


But we know that $\left\{\left(x_{n}, u_{n}\right)\right\}_{n \geqq 1}$ is relatively sequentially compact in $C(T, H) \times L^{2}(T, H)_{w}$. So by passing to a subsequence if necessary, we may assume that $x_{n} \rightarrow x$ in $C(T, H)$ and $u_{n} \stackrel{w}{\rightarrow} u \in M$ in $L_{m}^{2}(T)$. We claim that $(x, u)$ is an admissible pair. To this end note that for all $n \leqq 1$, we have:

$$
x_{n}(t)=S(t) x_{0}+\int_{0}^{t} S(t-s) w\left(s, x_{n}(s), u_{n}(s)\right) d s .
$$

From the properties of $w(\cdot, \cdot, \cdot)$, its linearity in $u$ and through the monotone convergence theorem, in the limit as $n \rightarrow \infty$, we get:

$$
\begin{aligned}
& x(t)=S(t) x_{0}+\int_{0}^{t} S(t-s) w(s, x(s), u(s)) d s, x(t) \in K(t) \\
& \Rightarrow(x, u) \text { is an admissible pair. }
\end{aligned}
$$

Finally from the properties of $\hat{h}(\cdot, \cdot, \cdot)$ and since $M \in P_{w k}\left(L^{2}(W)\right)$, we have

$$
m(0) \leqq J(x, u) \leqq \underline{\lim } J\left(x_{n}, u_{n}\right) \leqq \underline{\lim } m\left(\varepsilon_{n}\right) .
$$

Since $m\left(\boldsymbol{\varepsilon}_{n}\right) \leqq m(0) \Rightarrow m\left(\boldsymbol{\varepsilon}_{n}\right) \rightarrow m(0) \Rightarrow m(\cdot)$ is right continuous at $0 \Rightarrow$ the system is strongly calmand relaxable.

ACKNOWLEDGEMENT: The author would like to express his deep gratitude to the referee, for his many corrections, suggestions and remarks, that led to a considerable improvement of the material presented in this paper.

\section{REFERENCES}

[1] J.-P. Aubin And A. Cellina, Differential Inclusions, Springer, Berlin (1984).

[2] V. BARBU, Nonlinear Semigroups and Differential Equations in Banach Spaces, Noordhoff International Publishing, Leyden, The Netherlands (1976).

[3] C. Castaing and M. Valadier, Convex Analysis and Measurable Multifunctions, Lecture Notes in Math., Vol. 580, Springer, Berlin (1977).

[4] L. Cesari, Optimization-Theory and Applications, Springer, New York (1983).

[5] P.V. CHuong, Some results on density of extreme selections for measurable multifunctions, Math. Nachrichten 126 (1986), 311-326.

[6] F.H. ClARKE, Admissible relaxation in variational and control problems, J. Math. Anal. Appl. 51 (1975), 557-576.

[7] I. Ekeland and R. Temam, Convex Analysis and Variational Problems, North Holland, Amsterdam (1976).

[8] G. EDGAR, Measurability in a Banach space II, Indiana Univ. Math. Jour. 38 (1979), 559-579.

[9] A. FRYSZKowski, Continuous selections for a class of nonconvex multivalued maps, Studia Math. 76 (1983), 163-174.

[10] F. HIAI AND H. UMEGAKI, Integrals, conditional expectations and martingales of multivalued functions, J. Multiv. Anal. 7 (1977), 149-182.

[11] M. Kisielewicz, Multivalued differential equations in separable Banach spaces. 
J. Optim. Theory Appl. 37 (1982), 231-249.

[12] J.-L. Lions, Optimal Control of Systems Governed by Partial Differential Equations, Springer, New York (1971).

[13] N.S. Papageorgiou, Decomposable sets in the Lebesque-Bochner spaces, Comm. Math. Univ. S. P. 37 (1988), 49-62.

[14] N.S. PApageorgiou, Existence of solutions for differential inclusions with nonconvex right hand side, Intern. J. Math. and Math. Sci. 9 (1986), 459-469.

[15] N.S. PAPAgeorgiou, On multivaled evolution equations and differential inclusions in Banach spaces, Comm. Math. Univ. S. P. 36 (1987), 21-39.

[16] N.S. PAPAGEORgIoU, Convergence theorem for Banach space valued integrable multifunctions, Intern. Jour. Math. and Math. Sci. 10 (1987), 433-442.

[17] N.S. Papageorgiou AND E. Avgerinos, Viable solutions for evolution inclusions, Radovi Mat. 5 (1989), 61-70.

[18] N. PAvel, Differential Equations, Flow Invariance and Applications, Pitman, Boston (1984).

[19] N. Pavel and I. VRabie, Equations d'evolution multivoques, C.R.A.S. Paris, t. 287 (1987), 315-317.

[20] M.-F. Saint Beuve, On the extension of Von Neumann-Aumann's theorem, J. Funct. Anal. 17 (1974), 112-129.

[21] M.-F. Saint Beuve, Une extension des theoremes de Novikov et d'Arsenin, Semin. d'Analyse Convexe, Vol. 11, Exposé 18, Montpellier (1981).

[22] H. TANABE, Equations of Evolution, Pitman, Boston (1979).

UNIVERSITY OF CALIFORNIA

1015 Department of Mathematics

Davis, CALIFornia 95616, U.S. A. 